\title{
PERCEPCIÓN DE ESTUDIANTES Y DOCENTES DE LA UNIVERSIDAD AUTÓNOMA DE BUCARAMANGA (UNAB) SOBRE EL CURSO EN LÍNEA DE INDUCCIÓN A LA MODALIDAD VIRTUAL
}

\author{
Lina Maria Osorio VALDÉS ${ }^{1}$ \\ Verónica Salina URBINA ${ }^{2}$ \\ Fernando Jorge Mortera GUTIÉRREZ ${ }^{3}$
}

RESUMEN: El presente artículo tiene como objetivo describir y reflexionar sobre la percepción de los estudiantes y docentes del curso en línea: "Inducción a la modalidad virtual", ofrecido a los nuevos estudiantes de los diferentes programas de UNAB Tecnológica (Colombia). Para ello se realizó un estudio cuantitativo, con un diseño de investigación no experimental transversal que incluyó una muestra de 37 estudiantes y 3 docentes; a quienes se aplicaron diferentes instrumentos de recolección de información diseñados para esta investigación ( 2 encuestas y 1 guía de análisis del curso). Los resultados indican que la mayoría de estudiantes y docentes encuentran dentro del curso el cumplimiento de aspectos relacionados con los componentes pedagógico, tecnológico y organizativo; y ubican algunos aspectos susceptibles de mejora relacionados con el diseño del curso (componente pedagógico), duración y espacio de tiempo en el que es implementado (componente organizativo) y servicio de consejería (componente tecnológico).

PALABRAS CLAVE: Educación a distancia. Inducción a la modalidad en línea. Tecnologías de la información y de las comunicaciones (TIC).

\section{INTRODUCCIÓN}

La inducción a la modalidad virtual, se convierte en un elemento crítico dentro del proceso de enseñanza-aprendizaje a distancia, es como lo manifiesta Unigarro (2004, p.175), es:

[...] la propedéutica que han de realizar maestros y estudiantes. Este proceso debe ayudar a los actores a esclarecer todo lo relativo al modelo educativo que van a abordar y a las características que lo distinguen de otras maneras de hacer educación. Es fundamental que ambas partes conozcan el sentido y la mecánica; así se aúnan voluntades y esfuerzos en la misma dirección.

La inducción, se convierte en un espacio que les permite a los participantes de la misma, conocer con detalle todo lo relacionado con la educación a distancia como son:

\footnotetext{
1 UNAB - Universidad Autónoma de Bucaramanga. Bucaramanga - Colombia. 68001000 losorio3@unab.edu.co

${ }^{2}$ Instituto Tecnológico de Monterrey. Programas em Educación, Humanidades y Ciencias Sociales. Monterrey - NL - México. 64849 - veronica.salinas@ tecvirtual.mx

${ }^{3}$ Instituto Tecnológico de Monterrey. Programas em Educación, Humanidades y Ciencias Sociales. Monterrey - NL - México. 64849 - fmortera@tecvirtual.mx
} 
el tipo de recursos, el funcionamiento de la plataforma en la cual están almacenados, las diferentes herramientas de comunicación sincrónicas y asincrónicas; comprender el rol del estudiante y del docente; desarrollar habilidades de autoestudio y manejo del tiempo, entre otros aspectos, que les permiten a estudiantes y docentes realizar un tránsito progresivo hacia la modalidad, evitando generar desaciertos, más aún cuando hay muchas cosas, para los que la presencialidad, no los ha preparado (UNIGARRO, 2004).

Para atender a la necesidad de preparar a los nuevos estudiantes para la modalidad virtual, la Universidad Autónoma de Bucaramanga UNAB, diseñó el curso de inducción a la misma, el cual ha venido siendo ofertado para todos los estudiantes de nuevo ingreso de los diferentes programas a distancia y especialmente los de UNAB Tecnológica, Facultad en donde en este curso, se ha tenido un alto índice de deserción y no aprobación.

Con el objetivo de tener conocer más sobre los proceso de inducción a la modalidad a distancia, se realizó una cuidadosa búsqueda sobre antecedentes alusivos a los procesos de inducción en esta modalidad, pero no se encontró información relevante, dado que la gran mayoría de investigaciones a nivel nacional e internacional relacionadas con la educación a distancia se centran en los logros que experimentan los estudiantes, los avances en el uso de plataformas, los estilos de aprendizaje, los roles dentro del proceso enseñanza-aprendizaje, entre otros (ORGANISTA; BACKHOFF, 2002) En contraste, son escasas las investigaciones que se encuentran sobre la percepción que tienen los estudiantes y/o docentes respecto a la inducción en la modalidad virtual en un curso en línea, olvidando que su opinión es importante y esencial para poder evaluar, actualizar y rediseñar el currículo virtual (COUSSEMENT, 1995).

No obstante, sobre lo que sí se encontró información, fue sobre cómo la presencia de las tecnologías de la información y las comunicaciones genera entre los estudiantes una percepción positiva frente a la educación a distancia, ya que resulta para ellos atractiva, motivante y les permite aprender de manera autónoma, a la vez que les ofrece el control de su aprendizaje (CHIECHER; DONOLO; RIMAUDO, 2005). Sin embargo, también implica, tanto para estudiantes, como para docentes, nuevos desafíos respecto a las maneras de participar y hacer educación, ya que en América Latina, la gran mayoría de estudiantes provienen de un modelo de educación tradicional, presencial y pasivo; en donde dependen en su totalidad de las decisiones tomadas por la 
escuela y por sus maestros de manera unilateral; y por su parte los maestros, acostumbrados a desempeñarse en la modalidad presencial, traen consigo prácticas no necesariamente compatibles con la modalidad virtual (UNIGARRO, 2004).

Este ejercicio de búsqueda permitió evidenciar cómo las tecnologías de la información y de las comunicaciones (TIC), son un instrumento de apoyo importante en el proceso de enseñanza-aprendizaje, gracias al infinito número de posibilidades que ofrecen para la búsqueda y acceso a la información, su representación, procesamiento y transmisión (COLL et al., 2008). Sin embargo, es la alianza entre tecnología y educación, la que trae consigo mejoras en aspectos cuantitativos (poder llegar a un mayor número de estudiantes ubicados en diferentes lugares, disminución de costos logísticos, mayor cantidad de alternativas frente a canales y espacios de seguimiento y atención, etc.), así como en aspectos de carácter cualitativo (medios y recursos que enriquecen el proceso de aprendizaje, cercanía entre los diferentes actores del proceso a pesar de la distancia, posibilidad de atender a diferentes estilos y ritmos de aprendizaje, etc.) en la medida en que cada uno de ellos ofrece una riqueza de posibilidades para su proceso de aprendizaje.

\section{MARCO TEÓRICO}

A lo largo de esta investigación, la educación a distancia en línea o educación virtual es entendida como, la acción a través de la cual se generan espacios de formación, mediados por las TIC, en donde la comunicación entre los diferentes actores del proceso y su interés por iniciar el acto formativo y mantenerse en contacto con otros, son requisito para el alcance de los objetivos de formación (UNIGARRO, 2004), caracterizándose por permitir a los estudiantes acceder a ella sin límites de espacio o tiempo, dando la posibilidad de acceder a procesos de formación y recursos de acuerdo a las necesidades de quien desea aprender.

En el ambiente virtual de aprendizaje o a distancia, el docente cumple su papel de guía y orientador en la medida en que ofrece al estudiante herramientas y pistas que le ayudan a desarrollar su propio proceso de aprendizaje; y el estudiante por su parte, se convierte en uno de los elementos más importantes del proceso de formación, asumiendo un papel activo frente al mismo; para ello requiere de ciertas características, habilidades y competencias (autoestudio, gusto por la tecnología y por estar conectado, facilidades para comunicarse, disciplina, manejo del tiempo, trabajo en equipo, entre otros) que le permitan re-elaborar los contenidos que el ambiente virtual de aprendizaje 
le ofrece y poner en práctica sus habilidades de autoaprendizaje pues al ser él el centro del proceso de formación, es quien debe decidir qué, cómo, cuándo y dónde estudiar a partir de sus intereses (UNIGARRO, 2004).

Además de las características propias de un AVA (Ambiente Virtual de Aprendizaje), el docente y el estudiante como elementos claves, Salinas (2004) hace referencia a otros tres componentes: el pedagógico, el tecnológico y el organizativo, frente a los cuales se realizó el análisis del curso de Inducción a la modalidad virtual. El componente pedagógico describe cómo sucede el proceso de enseñanza-aprendizaje apoyado por las TIC a través de actividades de aprendizaje, materiales, interacción con el tutor y evaluación; el componente tecnológico, reseña la infraestructura tecnológica que se requiere para que suceda el proceso de enseñanza-aprendizaje apoyado por las TIC, acorde al modelo pedagógico; y el componente organizativo, relacionado con los aspectos que tienen que ver con la administración de la enseñanza-aprendizaje dentro del AVA, así como en la institución en general.

\section{EL PROCESO DE INDUCCIÓN}

La palabra inducción proviene del latín inductīo, que según la Real Académica de la Lengua Española (2001), es la acción y efecto de inducir (persuadir, instigar, ocasionar). Al hablar de un curso de inducción se hace referencia a una enseñanza preparatoria o propedéutica para el estudio de una disciplina.

Un curso de inducción debe ofrecer un acercamiento preliminar a los actores involucrados (docentes y estudiantes) frente a aspectos relacionados con el curso real tales como: el uso de la plataforma en la cual está alojado el curso, el modelo pedagógico y la modalidad virtual. La inducción no puede quedarse solamente en conocer el software y el hardware necesario, es importante que quien acceda a ella se apropie realmente de la propuesta educativa; para esto se proponen dos momentos importantes dentro de la inducción: la inducción a la modalidad virtual y la inducción a los programas y cursos (UNIGARRO, 2004).

La inducción a la modalidad virtual o en línea es el espacio en donde tanto docentes como estudiantes se enfrenten a preguntas como: ¿Cómo opera?, ¿Qué actividades se realizan?, ¿Qué la diferencia de la Educación presencial?, ¿Cuáles son las exigencias de la modalidad virtual en términos de tiempo? ¿Cuál es el rol del estudiante y del docente? Un buen proceso de inducción es necesario, dado que estudiantes y 
docentes provienen de una modalidad presencial, que no los ha preparado para abordar el proceso de enseñanza-aprendizaje desde una perspectiva en línea.

\section{PLANTEAMIENTO DEL PROBLEMA}

En América Latina, la gran mayoría de estudiantes provienen de un modelo de educación tradicional, presencial y pasivo, en donde dependen en su totalidad de las decisiones tomadas por la escuela y por sus docentes. No diferente, es el caso de los docentes, quienes acostumbrados a desempeñarse en la modalidad presencial, traen consigo prácticas no siempre compatibles con la modalidad a distancia (UNIGARRO, 2004). Es así, como la inducción a esta modalidad se convierte en un paso necesario, que busca que los estudiantes, comprendan el modelo educativo a distancia y los roles que desempeñarán al interior del mismo.

Es así como para este momento, y luego de varios años de implementación (2009-2012) del curso de inducción, se vio la necesidad de revisar su impacto educativo entre los estudiantes y docentes, por lo cual se procedió a hacer un primer sondeo.

Este ejercicio se planteó, en respuesta de los resultados alarmantes, producto de la última implementación del curso, en donde de 201 participantes inscritos, sólo 71 ingresaron al curso, 64 fueron finalmente atendidos y sólo 30 lo aprobaron. Es decir, el $25 \%$ de los estudiantes inscritos nunca ingresaron a la plataforma y del $75 \%$ restante, sólo el 50\% accedió a la misma. De ahí el interés de este estudio donde se pretende investigar a qué se pudiera deber esto, y se consideran aspectos precisos que pueden describir ideas de la percepción de los estudiantes y profesores relacionadas con el diseño del curso, rol del profesor, la participación del estudiante (componente pedagógico); la modalidad a distancia y sus implicaciones administrativas y logísticas (componente administrativo); las tecnologías y su funcionamiento (componente tecnológico), entre otros. De esta manera a partir de ello, implementar mejoras, que ayuden a reducir las cifras anteriormente descritas y en general identificar fortalezas y áreas de oportunidad.

\section{MÉTODO}

La investigación realizada se abordó desde un enfoque cuantitativo, de tipo descriptivo. Específicamente se habla de una investigación cuantitativa de tipo descriptivo, dado que esta investigación tuvo como objetivo recoger información sobre la percepción de los estudiantes y docentes respecto al curso de inducción en los tres 
componentes analizados, más no establecer relaciones entre ellas (HERNÁNDEZ; FERNÁNDEZ; BAPTISTA, 2010).

Por su parte, el diseño utilizado para la recolección de información fue el no experimental transeccional (transversal). No experimental, en la medida en que se estudió una situación sucedida previamente y por lo tanto, ajena al control del investigador, en donde las variables no fueron manipuladas de manera intencional, sino que la situación objeto de estudio se revisó en su contexto natural para posteriormente ser analizada; y transeccional, puesto que el alcance final de la investigación fue de tipo descriptivo; para lo que se recolectaron datos en un momento único, buscando conocer la manera en que se hacían evidentes cada una de las variables en el público objetivo (HERNÁNDEZ; FERNÁNDEZ; BAPTISTA, 2010).

Para la recolección de los datos, se realizó un plan que permitiera al investigador reunir la información con un propósito (HERNÁNDEZ; FERNÁNDEZ; BAPTISTA, 2010), por lo que fue necesario identificar el tipo de muestra, técnicas o instrumentos más pertinentes y acordes con los objetivos de la investigación, el alcance y diseño de la misma. Para este estudio se determinó que la muestra fuese de tipo probabilístico, puesto que se buscaba hacer estimaciones de la manera en que se presentaron las variables en una población. Este tipo de muestra permite medir el tamaño del error con relación a las predicciones y ofrece a todos los sujetos de la población la misma posibilidad de ser elegidos como muestra (HERNÁNDEZ, FERNÁNDEZ; BAPTISTA, 2010).

La definición de la muestra se realizó utilizando un marco muestral, el cual correspondió a un listado de los 65 participantes inscritos en el curso de Inducción a la modalidad virtual (ver apéndice A en el documento original), de tal manera que fuese posible numerarlos para seleccionar la muestra. Paso seguido, se calculó el número de unidades muestrales requeridas para conformar una muestra de calidad utilizando el programa estadístico STATS, en donde su ubicaron los siguientes datos: Tamaño del universo (número total del estudiantes que participaron en el curso de inducción: 65), error máximo aceptable (porcentaje de error que se admite: 9\%) Porcentaje estimado de la muestra (representatividad de la muestra vs. no representatividad: se dejó por default la sugerida por el programa estadístico STATS: 50\%) y nivel deseado de confianza (complemento del error máximo aceptable: 90\%). 
Resultado de este ejercicio, el grupo muestra quedó constituido por 3 docentes y 37 estudiantes, los cuales fueron seleccionados teniendo en cuenta los siguientes criterios:

-Para el caso de los docentes: que se hubiesen desempeñado como tutores del curso de Inducción a la Modalidad virtual de UNAB Tecnológica durante el primer semestre (Marzo 13-26) de 2012 en la UNAB.

-Para el caso de los estudiantes: haber sido estudiantes de nuevo ingreso que tomaron y finalizaron el curso de Inducción a la modalidad virtual de UNAB Tecnológica durante el primer semestre (Marzo 13-26) de 2012 en la UNAB.

Definida la muestra, se diseñaron tres instrumentos (dos cuestionarios y una guía de análisis) construidos a partir de una tabla de triple entrada con el objetivo de tener claridad con relación a las variables o categorías contenidas en la pregunta de investigación, los cuales fueron diligenciados en línea a través de la herramienta Survey Monkey por los participantes. Los cuestionarios estaban dirigidos a docentes ${ }^{4}$ y estudiantes ${ }^{5}$.

La guía de análisis del curso (rejilla de observación con algunos criterios para navegar y ubicar información dentro del mismo) fue utilizada por la investigadora para facilitar la recolección de información sobre el curso, el cual se encuentra alojado en una plataforma Learning Management System (LMS): Blackboard, utilizado por la $\mathrm{UNAB}^{6}$.

Paso seguido, y con el objetivo de garantizar la confiabilidad y validez de los instrumentos diseñados, se llevó a cabo una prueba piloto intentando recrear la aplicación real de los diferentes instrumentos para ello se administraron los instrumentos de recolección de información a una pequeña muestra, con el objetivo de identificar la pertinencia, coherencia y eficacia de los mismos, así como las condiciones y procedimientos necesarios para su aplicación.

Finalmente, y luego de aplicados los diferentes instrumentos, se realizó la codificación del cuestionario dirigido a estudiantes para de esta forma realizar un ejercicio de análisis que permitiera la obtención de frecuencias relativas y porcentajes y el cálculo de estadísticas descriptivas como la media y la desviación estándar. Los datos recopilados del cuestionario de docentes y la guía de análisis del curso, fueron

\footnotetext{
${ }^{4}$ Disponible a: <http://es.surveymonkey.com/s/paradocentes>. Accès: 30 jan. 2014.

${ }^{5}$ Disponible a: <http://es.surveymonkey.com/s/encuestaMTE_estudiantes >. Accès: 30 jan. 2014.

${ }^{6}$ Disponible a: <http://castor.unab.edu.co/>. Accès: 30 jan. 2014.
} 
organizados, mediante un resumen descriptivo por pregunta. Los datos recopilados a través de los cuestionarios aplicados a los 3 docentes y de la guía de análisis del curso no recibieron un tratamiento estadístico especial, por lo tanto se recopiló la información en una matriz que permitió realizar un resumen descriptivo de las respuestas.

\section{RESULTADOS}

La presentación de resultados se realizó partir de la elaboración de un resumen para cada una de las preguntas y la obtención de frecuencias relativas y porcentajes por cada componente. Además se hicieron cálculos de estadísticas descriptivas como la media y la desviación estándar para cada pregunta. A continuación se presentan los resultados organizados por componentes (pedagógico, tecnológico y organizativo) y dentro de cada uno de ellos se clasifica la información de cada una de las variables atendiendo a los tres diferentes instrumentos de recolección de información.

Componente Pedagógico: Describe cómo sucede el proceso de enseñanzaaprendizaje apoyado por las TIC a través de actividades de aprendizaje, materiales, interacción con el tutor y evaluación (SALINAS, 2004). Para realizar el análisis del componente pedagógico en el curso de Inducción a la modalidad virtual, se utilizaron preguntas dirigidas a tres públicos diferentes, a través tres tipos de instrumentos que permitieron compilar información para determinar aquellos aspectos de este componente en los que estudiantes, docentes e investigadora coincidieron en encontrar en el curso de inducción y aquellos que son susceptibles de mejora.

Como resultado de este proceso de análisis y contraste de los resultados, se observa que en general los tres públicos consultados coinciden en varios de los aspectos, convirtiéndose éstos en áreas fuertes del componente pedagógico que en el interior del curso es posible evidenciar en (ver tabla 1):

- Presentación de actividades, metodología, instrucciones y objetivos.

- Sugerencia de la manera de distribuir el tiempo para el desarrollo de las actividades y las lecturas.

- Actividades que permiten la apropiación de herramientas de la plataforma y habilidades propias del rol del estudiante virtual.

- Actividades que promueven la interacción entre estudiantes-estudiantes y docentes-estudiantes. 
- Respuesta y retroalimentación o por parte del docente a las inquietudes de los participantes.

- Motivación a los participantes por parte del docente a través de diferentes estrategias (mensajes de correo individuales, anuncios, retroalimentación en el foro, etc.).

- Material de apoyo para docentes y estudiantes.

- Coherencia entre la estrategia evaluativa y los objetivos de aprendizaje.

Como aspectos a dar especial atención y que pueden ser susceptibles de mejora en este componente se encuentran:

- El acceso de los estudiantes a otros cursos virtuales, de manera previa a la participación en el curso de inducción a la modalidad virtual.

- Revisión del número de actividades vs. Tiempo para el desarrollo de las mismas.

- Presentación de las indicaciones para el desarrollo de las actividades con mayor detalle o ejemplificando.

- Flexibilidad de los tutores en el manejo de los tiempos (encontrar un punto medio).

- Alto número de actividades y lecturas, frente al número de días dispuesto para el desarrollo del curso. 
Tabla 1 - Integración de resultados del Componente pedagógico

\begin{tabular}{|c|c|c|c|c|}
\hline \multicolumn{5}{|c|}{ Componente Pedagógico } \\
\hline \multicolumn{5}{|c|}{ Percepción } \\
\hline $\begin{array}{l}\frac{\pi}{0} \\
\frac{0}{0} \\
\frac{0}{0} \\
\frac{0}{0}\end{array}$ & \begin{tabular}{l} 
Los estudiantes \\
consideran que el \\
\multicolumn{1}{c}{ curso } \\
Presenta indicaciones \\
Presenta los objetivos \\
generales del mismo \\
Sugiere una \\
distribución del \\
tiempo para \\
desarrollo.
\end{tabular} & \begin{tabular}{lr}
\multicolumn{2}{c}{ Los docentes } \\
consideran que el \\
\multicolumn{2}{c}{ curso: } \\
Presenta & la \\
metodología & para \\
su desarrollo & \\
Sugiere & una \\
distribución & del \\
tiempo & \\
Presenta & los \\
objetivos & y \\
duración del curso.
\end{tabular} & $\begin{array}{l}\text { La investigadora } \\
\text { considera que el } \\
\text { curso: } \\
\text { Presenta instrucciones } \\
\text { y metodología para su } \\
\text { desarrollo en la } \\
\text { herramienta } \\
\text { contenidos. } \\
\text { Presenta los objetivos } \\
\text { de aprendizaje por lo } \\
\text { que se propenden la } \\
\text { herramienta } \\
\text { contenidos. } \\
\text { Sugiere de } \\
\text { distribución } \\
\text { tiempo por semanas, } \\
\text { la cual se encuentra } \\
\text { ubicada en la } \\
\text { herramienta } \\
\text { contenidos. }\end{array}$ & $\begin{array}{l}\text { Aspectos susceptibles } \\
\text { de mejora } \\
\text { Evitar que los } \\
\text { estudiantes participen } \\
\text { de manera previa al } \\
\text { curso de inducción en } \\
\text { los cursos base del } \\
\text { programa. }\end{array}$ \\
\hline$\frac{\tilde{d}}{\frac{0}{0}}$ & $\begin{array}{l}\text { Posee actividades } \\
\text { agradables. } \\
\text { Sugiere actividades } \\
\text { que ayudan al alcance } \\
\text { de los objetivos de } \\
\text { aprendizaje. } \\
\text { Sugiere actividades } \\
\text { que favorecen el uso } \\
\text { de herramientas de la } \\
\text { plataforma y } \\
\text { favorecen las } \\
\text { habilidades propias } \\
\text { del rol del estudiante } \\
\text { virtual. } \\
\text { Tienen un número de } \\
\text { actividades suficiente, } \\
\text { Dispone del tiempo de } \\
\text { acuerdo a al número } \\
\text { de actividades } \\
\text { Presenta indicaciones } \\
\text { para desarrollo de las } \\
\text { actividades. } \\
\text { Promueve } \\
\text { interacción an entre } \\
\text { estudiantes } \\
\text { estudiantes } \\
\text { estudiantes -docentes. } \\
\text { Sugiere espacios para } \\
\text { que el docente realice } \\
\text { una retroalimentación } \\
\text { oportuna. }\end{array}$ & $\begin{array}{l}\text { Presenta } \\
\text { actividades que } \\
\text { promueven el logro } \\
\text { de los objetivos } \\
\text { Presenta } \\
\text { indicaciones para el } \\
\text { desarrollo de las } \\
\text { actividades } \\
\text { Maneja tiempos } \\
\text { acordes al número } \\
\text { de actividades. }\end{array}$ & $\begin{array}{l}\text { Presenta actividades } \\
\text { que promueven el uso } \\
\text { de la plataforma y } \\
\text { actividades que } \\
\text { buscan apoyar al } \\
\text { estudiante en la } \\
\text { construcción de su rol } \\
\text { como estudiante } \\
\text { virtual. } \\
\text { Posee } 12 \text { actividades } \\
\text { y } 7 \text { lecturas para ser } \\
\text { desarrolladas en } 14 \\
\text { días. }\end{array}$ & $\begin{array}{l}\text { Presentación de las } \\
\text { indicaciones para el } \\
\text { desarrollo de las } \\
\text { actividades con } \\
\text { mayor detalle u } \\
\text { ofreciendo ejemplos. } \\
\text { Flexibilidad de los } \\
\text { tutores frente a la } \\
\text { entrega de actividades } \\
\text { y manejo de los } \\
\text { tiempos. } \\
\text { Alto número de } \\
\text { actividades y lecturas, } \\
\text { frente al número de } \\
\text { días para el desarrollo } \\
\text { del curso. }\end{array}$ \\
\hline
\end{tabular}




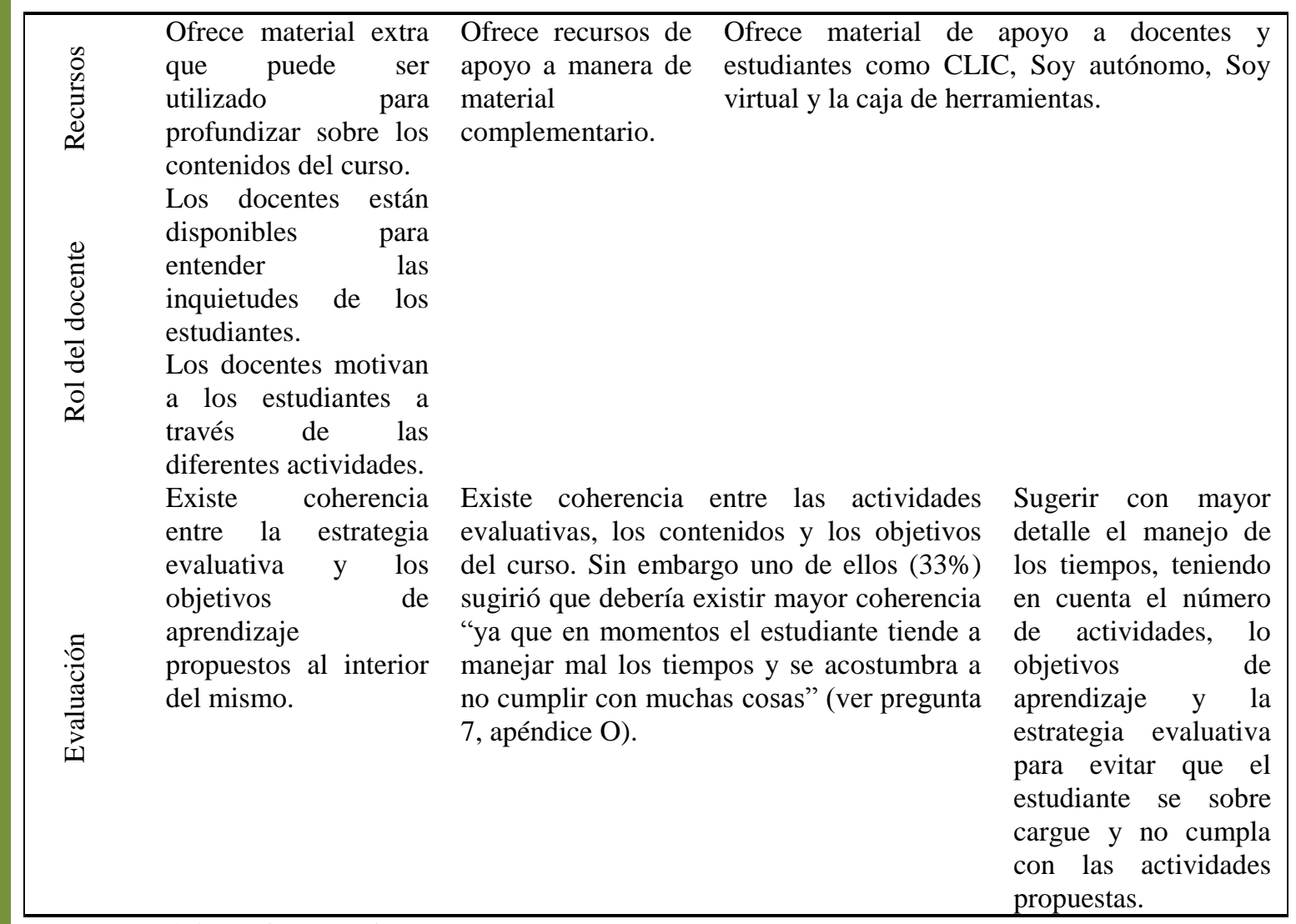

Fuente: Elaboración propia.

Componente Tecnológico: se centra en los procesos de interacción y comunicación al interior del curso y se caracteriza por generar los espacios y recursos necesarios para que la interacción entre los diferentes actores del proceso sea posible (DUART; LUPIAÑÉZ, 2005). En general los tres públicos consultados coinciden en varios de los aspectos, convirtiéndose estos en áreas fuertes del componente tecnológico que en el interior del curso es posible evidenciar en (ver tabla 2):

- Recursos de apoyo como manuales y video tutoriales.

- Acceso al servicio de consejería.

- Facilidad para acceder a diferentes fuentes de información.

- Interfaz homogénea, consistente, reversible y en general fácil de navegar a través de cualquier dispositivo y desde cualquier lugar.

- Herramientas para promover el trabajo colaborativo tales como chat, foros, blog, etc.

Como aspectos a dar especial atención y que pueden ser susceptibles de mejora en este componente se encuentran: 
- Dar a conocer a los estudiantes las funciones y utilidad del servicio de consejería, buscando aumentar el uso de este servicio como apoyo a su proceso como estudiantes virtuales.

- Promover el uso del chat como herramienta que facilita el trabajo colaborativo.

- Hacer evidente al interior de la plataforma la ubicación del acceso a los manuales y material de apoyo.

Tabla 2 - Integración de resultados del Componente Tecnológico

\begin{tabular}{|c|c|c|c|c|}
\hline \multicolumn{5}{|c|}{ Componente Tecnológico } \\
\hline \multicolumn{5}{|c|}{ Percepción } \\
\hline Aspecto & $\begin{array}{c}\text { Los estudiantes } \\
\text { consideran que el } \\
\text { curso }\end{array}$ & $\begin{array}{c}\text { Los docentes } \\
\text { consideran que el } \\
\text { curso: }\end{array}$ & $\begin{array}{c}\text { La investigadora considera } \\
\text { que el curso: }\end{array}$ & $\begin{array}{c}\text { Aspectos susceptibles } \\
\text { de mejora }\end{array}$ \\
\hline 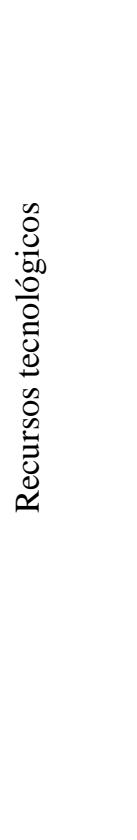 & \begin{tabular}{l} 
Ofrece diferentes \\
recursos de apoyo y \\
canales de \\
comunicación tales \\
como los manuales \\
y videotutoriales. \\
\multicolumn{1}{c}{ Ofrece el } \\
servicio de \\
consejería.
\end{tabular} & $\begin{array}{lr}\text { Las } & \text { diferentes } \\
\text { herramientas } & \\
\text { ofrecidas } & \text { al } \\
\text { interior del } & \text { curso } \\
\text { favorecen } & \text { la } \\
\text { interacción } & \text { entre } \\
\text { los } & \text { diferentes } \\
\text { actores } & \text { del } \\
\text { proceso. } & \\
\text { Ofrece } & \text { manuales } \\
\text { útiles } & \text { para } \\
\text { despejar dudas de } \\
\text { los estudiantes } \\
\text { frente a recursos } \\
\text { de la plataforma y } \\
\text { software } \\
\text { Ofrece el servicio } \\
\text { de consejería el } \\
\text { cual es de gran } \\
\text { utilidad. }\end{array}$ & $\begin{array}{l}\text { Ofrece herramientas que } \\
\text { favorecen la interacción y el } \\
\text { trabajo colaborativo tales } \\
\text { como foros, grupos, chat y } \\
\text { BB Collaborate. } \\
\text { Carga rápidamente } \\
\text { independientemente del } \\
\text { dispositivo en el que se } \\
\text { visualice. el acceso a } \\
\text { Permite el a } \\
\text { diferentes manuales y } \\
\text { videotutoriales para el uso } \\
\text { de Blackboard y la descarga } \\
\text { de software. }\end{array}$ & 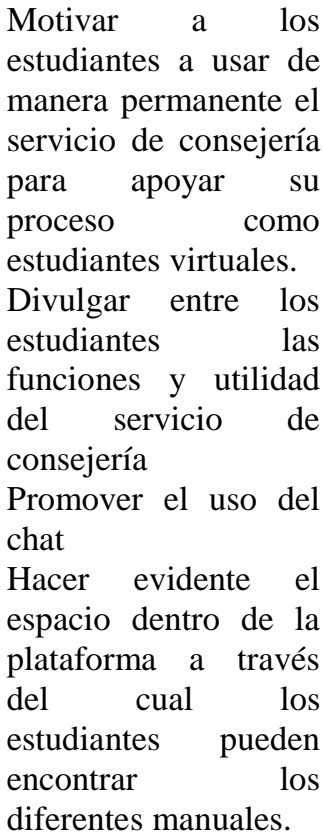 \\
\hline 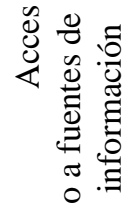 & $\begin{array}{l}\text { Permite el acceso a } \\
\text { diferentes fuentes } \\
\text { de información } \\
\text { desde el interior del } \\
\text { curso virtual. }\end{array}$ & & $\begin{array}{l}\text { Permite acceder a fuentes de } \\
\text { información a través de } \\
\text { CLIC, herramienta en la que } \\
\text { se compilan manuales, tips, } \\
\text { descarga de software, etc. }\end{array}$ & $\begin{array}{lr}\text { Hacer explícito en la } \\
\text { plataforma } & \text { la } \\
\text { funcionalidad } & \text { de } \\
\text { CLIC } & \text { como } \\
\text { herramienta. } & \end{array}$ \\
\hline 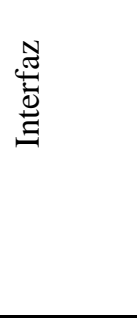 & $\begin{array}{l}\text { Presenta una } \\
\text { interfaz amigable y } \\
\text { fácil de navegar. } \\
\text { Permite el acceso } \\
\text { desde cualquier } \\
\text { lugar y a través de } \\
\text { cualquier } \\
\text { dispositivo. }\end{array}$ & $\begin{array}{l}\text { Posee una interfaz } \\
\text { agradable }\end{array}$ & $\begin{array}{l}\text { Ofrece a sus usuario } \\
\text { de fácil acceso y navegación } \\
\text { ofrece banners personalizado } \\
\text { colores que permite al es } \\
\text { (menú principal del curso), e } \\
\text { diferentes dispositivos ajustá } \\
\text { pantalla y está alojado en } \\
\text { como lo es BlackBoard. }\end{array}$ & $\begin{array}{l}\text { una interfaz amigable, } \\
\text { en la medida en que } \\
\text { maneja una gama de } \\
\text { tudiantes personalizar } \\
\text { s accesible a través de } \\
\text { ndose al tamaño de la } \\
\text { ina plataforma estable }\end{array}$ \\
\hline
\end{tabular}

Fuente: Elaboración propia. 
Componente Organizativo: hace referencia a la disposición de los diferentes canales de comunicación al interior del curso, pero sobre todo a su administración, en la medida en que se realiza una selección de los más apropiados y se promueve el uso de los mismos para generar espacios de trabajo colaborativo. En esencia el componente organizativo tiene como función administrar, organizar y disponer de los diferentes elementos del curso virtual (LUKER, 2000). Las siguientes son áreas fuertes del componente organizativo que en el interior del curso es posible evidenciar en (ver tabla 3):

- Material previo que permite al estudiante conocer la finalidad del curso y la manera de acceder a él.

- Publicación de recursos de apoyo en la medida en que el estudiante manifiesta su necesidad de uso.

- Disposición de herramientas medios y canales para promover el trabajo colaborativo y la comunicación.

- Retroalimentación por parte del docente respecto a las diferentes actividades.

Como aspectos a dar especial atención y que pueden ser susceptibles de mejora se encuentran:

- Momento durante el semestre en el que es tomado el curso por los estudiantes.

- Duración del curso vs. Número de actividades y contenidos. 
Tabla 3 - Integración de resultados del Componente Organizativo

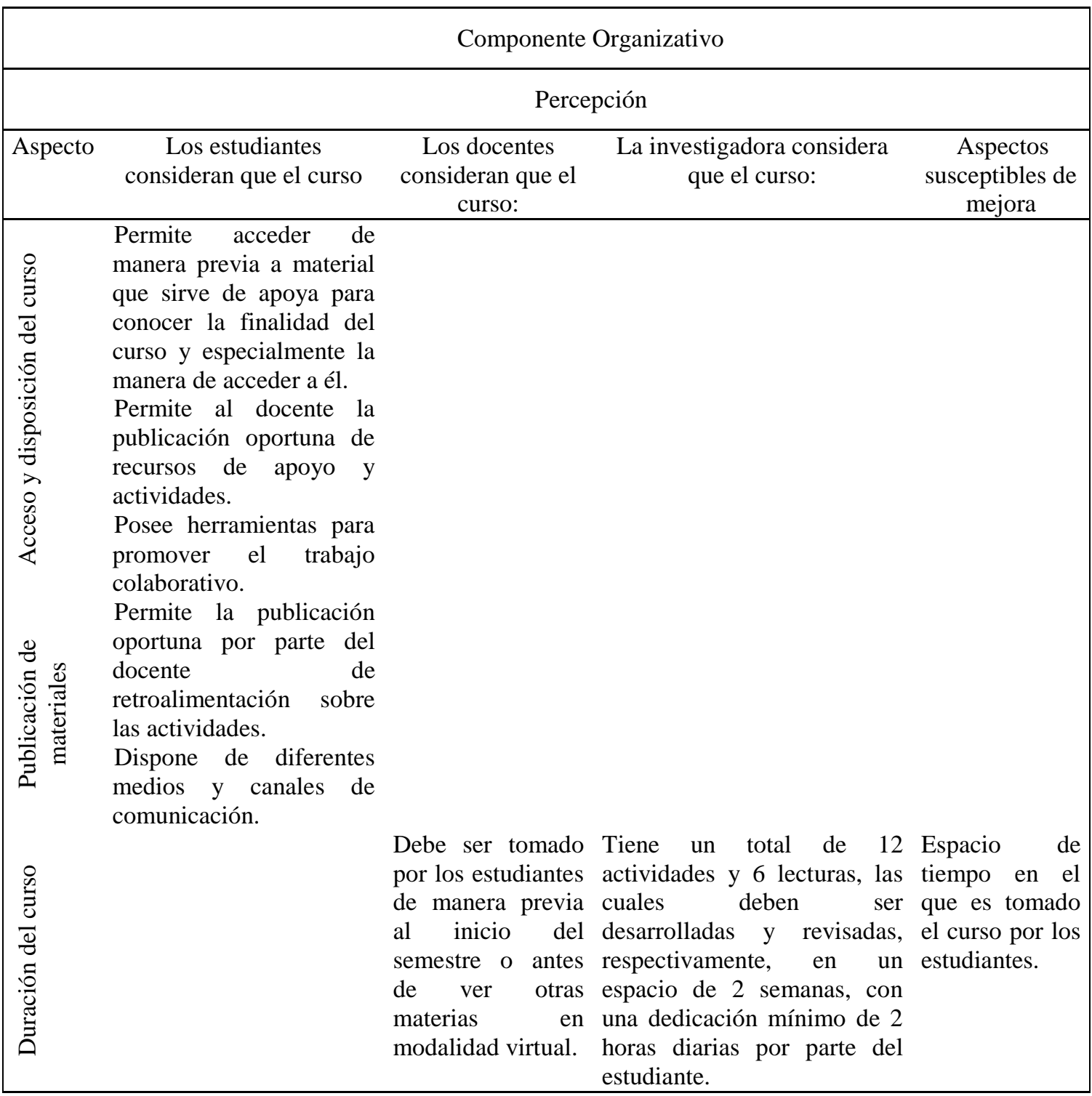

Fuente: Elaboración propia.

\section{DISCUSIÓN}

El proceso de inducción a la educación a distancia es de vital importancia en la medida en que la mayoría de los estudiantes que actualmente están accediendo a esta modalidad provienen de un proceso de enseñanza presencial. Para el caso del curso analizado a lo largo de esta investigación, tanto estudiantes como docentes, ubicaron dentro del mismo características propias de los componentes pedagógico, tecnológico y organizativo de un ambiente virtual de aprendizaje; dentro de las cuales se resaltaron como fortalezas: la claridad en la metodología, la variedad en las actividades y recursos, la posibilidad de interacción entre docentes y estudiantes, la coherencia entre la 
evaluación, contenidos y actividades de aprendizaje, el servicio de acompañamiento y apoyo a través de consejería, la facilidad de navegación, entre otros aspectos.

No obstante, también se identificaron algunos elementos susceptibles de mejora relacionados con la fecha de inicio del curso, el número de actividades vs. el número de semanas de duración, la homogeneidad en los criterios para las evaluaciones, tiempos de entrega, etc. manejados por los docentes, la posibilidad de ampliación por parte de los docentes de las indicaciones para el desarrollo de las actividades, el fomento del uso del servicio de Consejería (sólo el $27 \%$ de los estudiantes encuestados utilizan este servicio), entre otros.

Igualmente, producto del ejercicio investigativo surgen una serie de aspectos que se convierten en oportunidades para futuras investigaciones; como ubicar las actividades de mayor preferencia para los estudiantes virtuales y determinar el tiempo real que toma a los estudiantes la revisión de los contenidos dispuestos en línea, para el caso del componente pedagógico.

Por su parte, desde el componente tecnológico los resultados obtenidos frente al uso de los servicios de consejería se convierten en una oportunidad para investigar la efectividad, funcionalidad y percepción que tienen los estudiantes frente a este servicio, así como su influencia en el rendimiento y motivación de los mismos. Desde el componente organizativo, el estudio de los diversos canales de comunicación, se convierte en una oportunidad para ampliar la oferta de herramientas y conocer cuáles son más cómodas para comunicarse y construir con otros.

Esta investigación, se convierte en un primer paso para continuar indagando sobre la importancia de la inducción en los procesos de enseñanza a distancia, la mejor manera de realizarla y los diferentes componentes que hacen parte de una ambiente virtual de aprendizaje y que garantizan que se dé una dinámica entre los diferentes actores a través del mismo.

\section{UNIVERSIDAD AUTÓNOMA DE BUCARAMANGA STUDENTS AND FACULTY'S PERCEPTION ABOUT THEIR EXPERIENCE ON AN ONLINE COURSE WHICH INTRODUCE THEM TO DISTANCE EDUCATION ISSUES}

$\overline{\text { ABSTRACT: } \text { The present article will describe and reflect the perceptions of students }}$ and faculty about their educational experience from one online course, named: "Induction to a virtual mode education". This course was offered to new students from different programs from a Colombian higher education institution (UNAB). A 
quantitative study was conducted with a transversal no experimental research design that included a sample of 37 students and 3 teachers. Different instruments for data gather were designed for this research study (2 surveys -questionnaires- and one analysis course guide). The results indicate that the majority of students and faculty perceive that the course accomplish successfully different issues related to pedagogical , technological and organizational components of the course design; several areas for improvement related to the design of the course (educational component), and duration of time in which it is implemented (organizational component) and counseling (online) were detected, in this article will be described and commented.

KEYWORDS: Distance education. Introduction to distance learning. Information and communication technologies (ICT).

\section{REFERENCIAS}

CHIECHER, A.; DONOLO, D.; RINAUDO, M. C. Percepciones del aprendizaje en contextos presenciales y virtuales. La perspectiva de alumnos universitarios. RED: Revista de Educación a Distancia, v.5, n.13, p.2-3, 2005. Disponible a: <http://redalyc.uaemex.mx/redalyc/html/547/54701302/54701302_1.html>. Accès: 20 feb. 2014.

COLL, C. et al. Psicología de la educación virtual: aprender y enseñar con las tecnologías de la información y la comunicación. Madrid: Ediciones Morata, 2008.

COUSSEMENT, S. H. Educational telecommunication: Does it work? Tuscaloosa: University of Alabama, 1995.

DUART, J.; SANGRÀ, A. Desarrollo organizativo del e-learning: procesos institucionales de desarrollo y apoyo a la formación: La gestión del e-learning. Barcelona: Universidad Oberta de Catalunya, 2005.

\section{HERNÁNDEZ, R.; FERNÁNDEZ, C. ,BAPTISTA, M. Metodología de la} investigación. México: McGrawHill, 2010.

LUKER, M. Preparing your campus for a networked future: educause leadership strategies. San Francisco: Jossey-Bass, 2000.

ORGANISTA, J.; BACKHOFF, E. Opinión de estudiantes sobre el uso de apoyos didácticos en línea en un curso universitario. Revista Electrónica de Investigación Educativa, Ensenada, v.4, n.1, p.1-14, 2002. Disponible a:

<file:///C:/Users/bibpat/Downloads/52-250-1-PB.pdf>. Accès: 20 feb. 2014.

SALINAS, J. Hacia un modelo de educación flexible: elementos y reflexiones. En: MARTÍNEZ, F.; PRENDES, M. P. (Coord.). Nuevas tecnologías y educación. Madrid: Pearson-Prentice Hall, 2004. p.145-150.

UNIGARRO, M. Educación virtual: encuentro educativo en el ciberespacio. Bucaramanga: Ed. da UNAB, 2004. 